\title{
Conversion to Combined Mycophenolate Mofetil and Low-Dose Calcineurin Inhibitor Therapy for Renal Dysfunction in Liver Transplant Patients: Never Too Late?
}

\author{
Susanne Beckebaum • Vito R. Cicinnati
}

Published online: 4 November 2010

(c) The Author(s) 2010. This article is published with open access at Springerlink.com

A progressively increasing life expectancy after liver transplantation (LT) leads to previously underestimated long-term complications, in particular renal damage as well as metabolic and cardiovascular disease [1]. Although the etiology of kidney damage in LT patients may be multifactorial, calcineurin-inhibitor (CNI)-induced nephrotoxicity significantly contributes to the development of renal dysfunction after LT [2]. CNIs may cause acute and chronic nephrotoxicity, with the latter usually being associated with structural changes in the kidney [3]. Acute nephrotoxicity comprises vascular effects such as vasoconstriction of the afferent arterioles, an increased secretion of vasoconstrictor factors including endothelin and thromboxane, activation of the renin-angiotensin system (RAS), and reduction of vasodilatation factors such as nitric oxide, prostacyclin and prostaglandin E2. Acute CNI-induced nephrotoxicity may further lead to tubular damage and/or dysfunction and thrombotic microangiopathy. Chronic CNI-related nephrotoxicity including arteriolar hyalinosis, tubular atrophy, interstitial fibrosis, and glomerular sclerosis is observed in most LT recipients with long-term CNI therapy [2]. Chronic CNI-related renal damage is thought to be a consequence of both CNIinduced hemodynamic changes and direct toxic effects on renal tubular epithelium. Despite the fact that chronic CNIrelated nephrotoxicity is associated with parenchymal

S. Beckebaum · V. R. Cicinnati

Department of Gastroenterology and Hepatology, University

Hospital Essen, University of Duisburg-Essen, Essen, Germany

S. Beckebaum $(\bowtie) \cdot$ V. R. Cicinnati

Department of General, Visceral and Transplantation Surgery,

University Hospital Essen, University of Duisburg-Essen, Essen, Germany

e-mail: susanne.beckebaum@uni-essen.de damage, various clinical studies have shown that CNI dose reduction or discontinuation results in an improvement of renal function in the majority of LT patients, thus suggesting a partly dose-dependent nephrotoxic effect and reversible functional kidney impairment [4]. In addition, CNI reduction or withdrawal is also associated with decreased cardiovascular risk in LT patients [5].

CNI sparing protocols in LT patients are usually based on introduction of mycophenolate mofetil (MMF) or mammalian target of rapamycin (mTOR) inhibitors (e.g. sirolimus and everolimus) into the immunosuppressive regimen. While the latter drug group is still awaiting approval for clinical use in LT patients, MMF has been has been clinically established since 1995 . MMF is a morpholinoethyl ester prodrug of mycophenolic acid (MPA), an inhibitor of inositol-monophosphate dehydrogenase that catalyzes the rate limiting step in de novo purine biosynthesis, thereby specifically suppressing the proliferation of $\mathrm{T}$ and $\mathrm{B}$ lymphocytes. MMF virtually lacks drug-related nephrotoxicity and does not increase cardiovascular risk. Complete replacement of CNI with MMF though bears an increased risk of acute rejection; although, combined MMF and low dose CNI therapy has been shown to be safe within this regard in several studies [4]. Gastrointestinal (e.g. diarrhea) and hematological (e.g. anemia and neutropenia) disorders are the most important and frequent side effects associated with MMF therapy, but they seldom require drug discontinuation [6].

Pageaux et al. [7] were the first to report results of a prospective randomized trial in LT patients comparing a combined low-dose CNI and MMF regimen with CNI monotherapy. Median time after LT was approximately 60 months in both groups at start of the trial. In the MMF group, CNI doses were reduced by at least $50 \%$ regardless of the baseline titer, whereas in MMF-free controls CNI 
doses could be reduced up to $25 \%$ of the initial dose. As compared to the control group, in the MMF/CNI group a significant amelioration of renal function became evident. In a more recent study from our group [8], LT patients with renal dysfunction were randomized either to receive MMF followed by stepwise reduction of CNI with defined minimal CNI-trough levels (MMF group), or to continue their maintenance CNI dose (control group). Time between LT and enrollment of patients into the study ranged from 12 to 199 months with a mean of 60 months. In our study, CNI dose was progressively tapered to achieve defined target trough levels as low as $2-4 \mathrm{ng} / \mathrm{ml}$ for tacrolimus and $25-50 \mathrm{ng} / \mathrm{ml}$ for cyclosporine A. In the MMF group renal function improved in $62 \%$ of patients, remained stable in $36 \%$ and deteriorated in only $2 \%$ after 12 months compared with baseline values. In the control group renal function tended to deteriorate during the study period. Of all tested variables, younger age at study entry was the only predictive factor for renal improvement in the MMF group. This, however, might simply reflect an increase in atherosclerotic vascular disease with older age.

Some studies have demonstrated that MMF directly exerts protective effects against inflammation and fibrosis progression. Suggested protective mechanisms include the inhibition of adhesion molecules involved in the migration of immune cells towards the allograft, reduced nitric oxide production with subsequent suppressed allograft injury via interactions with superoxides, elimination of antigenspecific $\mathrm{T}$ cells and reduced inflammatory cytokine synthesis [9]. MMF in combination with CNI taper has been shown to ameliorate fibrosis progression, graft inflammation and aminotransferase levels in LT patients with chronic hepatitis $\mathrm{C}$ virus (HCV) infection [10]. In a retrospective study including 168 LT recipients with chronic $\mathrm{HCV}$ infection, our group showed that long-term MMF is associated with superior graft and patient survival rates compared with patients who received no or short-term ( $<6$ months) MMF immunosuppressive regimens [11].

Several studies comprising immune and nonimmunemediated renal disease have provided evidence that MMF is effective in reversing structural changes in the kidney. CNI-associated RAS activation promotes renal interstitial fibrosis due to inhibition of matrix-metalloproteinase (MMP) activity leading to enhanced extracellular matrix accumulation [3]. While most of the favorable renal effects observed in CNI minimization protocols have been attributed to the lower or absent CNI exposure, there is some evidence that MMF may be directly nephroprotective. For instance, MPA has been shown in vitro to transcriptionally inhibit collagen expression, enhance the expression of MMP-1 and to modify the migratory and functional properties of fibroblasts, thus directly exerting antifibrotic activities [12]. In an animal model MPA has been demonstrated to significantly reduce glomerular and interstitial injury with pronounced decreases in myofibroblast infiltration and collagen accumulation [13]. A potential anti-atherosclerotic effect due to the immunomodulatory function of MMF has also been proposed [14]. In a clinical study with pancreas-kidney recipients, Nankivell et al. [15] showed that MMF treatment was associated with notable reduction in tubulo-interstitial, striped and periglomerular fibrosis, glomerulosclerosis, mesangial matrix deposition and arteriolar hyalinosis. In a more recent retrospective study, Karie-Guigues et al. [16] indicated that introduction of MMF without decreasing the CNI dose was associated with an improvement of renal function in the long term. There is thus accumulating evidence that MMF may directly exert nephroprotective effects. To date, however, it is not clear in which patients CNI-related renal dysfunction is reversible and in which morphological changes persist despite reduction or withdrawal of CNI. In addition, published data about the impact of conversion to MMF and low-dose CNI on long-term renal function and patient survival are still limited [17, 18]. Recently, Kornberg et al. [19] addressed this issue in a prospective study with 63 LT patients with CNI-induced renal dysfunction. The authors showed that at 60 months post-conversion, mean creatinine levels had significantly declined and mean glomerular filtration rate had significantly increased. Nearly half of the converted patients had CNI-related renal dysfunction for more than 2 years. Sustained renal response was observed in $73.1 \%$ of patients; full-dose MMF medication and early conversion were identified as independent predictors of persistent renal function improvement. Sustained renal response after drug conversion was the most relevant independent promoter of long-term survival in patients receiving MMF therapy. Although the study is flawed by the absence of a comparable control group of patients receiving CNI monotherapy, the reported results intuitively suggest a favorable long-term outcome for LT patients receiving MMF-based immunosuppression.

Based on a review of the literature, combined low-dose CNI and MMF therapy appears to be safe with regard to incidence of allograft rejection [4]. However, individual risk estimation of acute rejection by genetic and/or immunological factors is not yet clinically established. There is some evidence indicating that CNI treatment reduces the percentages of circulating regulatory $\mathrm{T}$ cells (Treg) which play a pivotal role in establishment of immunologic allograft tolerance [20]. Treg function critically depends on calcineurindependent interleukin-2 production and CNI have been shown to interfere with Treg induction in a dose-dependent manner [21]. Two prospective clinical studies so far specifically investigated the immunological risk in LT patients receiving MMF therapy. Our group previously showed that the circulating cytotoxic $\mathrm{T}$ lymphocyte effector pool 
diminished, whereas the number of $\mathrm{CD} 4+\mathrm{CD} 25+$ Foxp3 + Treg significantly increased over time in LT patients receiving combined low-dose CNI and MMF therapy as compared to CNI monotherapy [8]. More recently, Demirkiran et al. [22] indicated that conversion from CNI to MMF therapy increases both percentage and CD25 expression of CD4 + CD25 + Foxp3 + Treg. These clinical findings suggest that introduction of MMF into the immunosuppressive regimen may promote immunological tolerance to allogeneic transplants.

In recent years, prospective controlled trials have established the clinical safety and efficacy of combined MMF and low-dose CNI therapy in LT patients, as documented by low incidence of allograft rejection, and progressive improvement in renal function as well as in cardiovascular risk factors [7, 8, 23, 24]. Although most enrolled patients suffered from chronic CNI-related nephrotoxicity for several years, renal function improved in a significant number of converted LT patients. Thus, improvement of CNI-related nephrotoxicity may still occur after long lasting CNI exposure. Tentative conversion to MMF-based immunosuppression should therefore be encouraged whenever CNI-related renal impairment is suspected in LT patients. In conclusion, there is emerging evidence that introduction of MMF into the immunosuppressive regimen may improve patient and graft survival after LT. Further prospective controlled studies are nevertheless needed to determine the therapeutic impact of MMF on outcome of LT patients in the long term.

Open Access This article is distributed under the terms of the Creative Commons Attribution Noncommercial License which permits any noncommercial use, distribution, and reproduction in any medium, provided the original author(s) and source are credited.

\section{References}

1. Sethi A, Stravitz RT. Review article: Medical management of the liver transplant recipient-A primer for non-transplant doctors. Aliment Pharmacol Ther. 2007;25:229-245.

2. Bahirwani R, Reddy KR. Outcomes after liver transplantation: chronic kidney disease. Liver Transpl. 2009;15(Suppl 2): S70-S74.

3. Naesens M, Kuypers DR, Sarwal M. Calcineurin inhibitor nephrotoxicity. Clin J Am Soc Nephrol. 2009;4:481-508.

4. Farkas SA, Schnitzbauer AA, Kirchner G, et al. Calcineurin inhibitor minimization protocols in liver transplantation. Transpl Int. 2009;22:49-60.

5. Mells G, Neuberger J. Reducing the risks of cardiovascular disease in liver allograft recipients. Transplantation. 2007;83:1141-1150.

6. Shipkova M, Armstrong VW, Oellerich M, Wieland E. Mycophenolate mofetil in organ transplantation: Focus on metabolism, safety and tolerability. Expert Opin Drug Metab Toxicol. 2005;1: 505-526.

7. Pageaux GP, Rostaing L, Calmus Y, et al. Mycophenolate mofetil in combination with reduction of calcineurin inhibitors for chronic renal dysfunction after liver transplantation. Liver Transpl. 2006;12:1755-1760.

8. Cicinnati VR, Yu Z, Klein CG, et al. Clinical trial: Switch to combined mycophenolate mofetil and minimal dose calcineurin inhibitor in stable liver transplant patients-Assessment of renal and allograft function, cardiovascular risk factors and immune monitoring. Aliment Pharmacol Ther. 2007;26:1195-1208.

9. Allison AC, Eugui EM. Mechanisms of action of mycophenolate mofetil in preventing acute and chronic allograft rejection. Transplantation. 2005;80:S181-S190.

10. Bahra M, Neumann UI, Jacob D, et al. MMF and calcineurin taper in recurrent hepatitis $\mathrm{C}$ after liver transplantation: Impact on histological course. Am J Transplant. 2005;5:406-411.

11. Iacob S, Cicinnati VR, Hilgard P, et al. Predictors of graft and patient survival in hepatitis $\mathrm{C}$ virus $(\mathrm{HCV})$ recipients: Model to predict HCV cirrhosis after liver transplantation. Transplantation. 2007;84:56-63.

12. Roos N, Poulalhon N, Farge D, et al. In vitro evidence for a direct anti-fibrotic role of the immunosupressive drug mycophenolate mofetil. J Pharmacol Exp Ther. 2007;321:583-589.

13. Badid C, Vincent M, McGregor B, et al. Mycophenolate mofetil reduces myofibroblast infiltration and collagen III deposition in rat remnant kidney. Kidney Int. 2000;58:51-61.

14. van Leuven SI, Kastelein JJ, Allison AC, Hayden MR, Stroes ES. Mycophenolate mofetil (MMF): Firing at the atherosclerotic plaque from different angles? Cardiovasc Res. 2006;69:341-347.

15. Nankivell BJ, Wavamunno MD, Borrows RJ, et al. Mycophenolate mofetil is associated with altered expression of chronic renal transplant histology. Am J Transplant. 2007;7:366-376.

16. Karie-Guigues S, Janus N, Saliba F, et al. Long-term renal function in liver transplant recipients and impact of immunosuppressive regimens (calcineurin inhibitors alone or in combination with mycophenolate mofetil): The TRY study. Liver Transpl. 2009;15:1083-1091.

17. Jain A, Vekatramanan R, Eghtesad B, et al. Long-term outcome of adding mycophenolate mofetil to tacrolimus for nephrotoxicity following liver transplantation. Transplantation. 2005;80: 859-864.

18. Biselli M, Vitale G, Gramenzi A, et al. Two yr mycophenolate mofetil plus low-dose calcineurin inhibitor for renal dysfunction after liver transplant. Clin Transplant. 2009;23:191-198.

19. Kornberg A, Küpper B, Thrum K, et al. Sustained renal response to mycophenolate mofetil and CNI taper promotes survival in liver transplant patients with CNI-related renal dysfunction. Dig Dis Sci. 2010. doi:10.1007/s10620-010-1386-z.

20. Wood KJ, Sakaguchi S. Regulatory T cells in transplantation tolerance. Nat Rev Immunol. 2003;3:199-210.

21. Zeiser R, Nguyen VH, Beilhack A, et al. Inhibition of CD4 + $\mathrm{CD} 25$ + regulatory $\mathrm{T}$-cell function by calcineurin-dependent interleukin-2 production. Blood. 2006;108:390-399.

22. Demirkiran A, Sewgobind VD, van der Weijde J, et al. Conversion from calcineurin inhibitor to mycophenolate mofetil-based immunosuppression changes the frequency and phenotype of CD4 + FOXP3 + regulatory T cells. Transplantation. 2009;87: 1062-1068.

23. Kornberg A, Kupper B, Hommann M, Scheele J. Introduction of MMF in conjunction with stepwise reduction of calcineurin inhibitor in stable liver transplant patients with renal dysfunction. Int Immunopharmacol. 2005;5:141-146.

24. Gerhardt T, Terjung B, Knipper P, et al. Renal impairment after liver transplantation-A pilot trial of calcineurin inhibitor-free vs. calcineurin inhibitor sparing immunosuppression in patients with mildly impaired renal function after liver transplantation. Eur J Med Res. 2009;14:210-215. 